\title{
Complex resistance of spring bread wheat lines to biotic and abiotic stress
}

Lapochkina I. ${ }^{1 *}$, Gainullin N. ${ }^{1}$, Baranova O. ${ }^{2}$, Kovalenko N. ${ }^{2}$, Marchenkova L. ${ }^{1}$

${ }^{1}$ Federal Research Center "Nemchinovka", Odinzovo, Russia

${ }^{2}$ All-Russian Institute for Plant Protection, St. Petersburg-Pushkin, Russia

*email: inna-lapochkina@yandex.ru

The original initial material of spring bread wheat with group resistance to stem and leaf rust was developed using new sources of stem rust resistance: winter common wheat GT 96/90 (Bulgaria) and line 119/4-06rw with genetic material, respectively, of T. migushovae and (Ae. speltoides and S. cereale), spring wheat line 113/00i-4 obtained using the species Ae. triuncialis and T. kiharae, as well as spring accession 145/00i with genetic material of Ae. speltoides. The transfer of effective $\mathrm{Sr}$ genes to progeny was monitored using molecular markers. The new lines underwent a field assessment of resistance to leaf and stem rust in the epiphytotic development of diseases in the Central region of the RF, as well as in the North Caucasus and Western Siberia and showed high resistance to these pathogens with different racial composition. Eleven genotypes with group resistance to these diseases and the parental forms that took part in the origin of the lines were assessed for resistance to spot blotch (Cochliobolus sativus) and tan spot (Pyrenophora tritici-repentis) using isolates from Kazakhstan and Omsk under laboratory conditions. Highly resistant parental form of winter bread wheat from the collection "Arsenal" 119/4-06rw (wheat-Ae. speltoides-rye hybrid $2 n=42$ ) was isolated simultaneously to two spots and four medium-resistant genotypes to both populations of tan spot (Kazakhstan and Omsk), as well as genotypes resistant to the Omsk population of $P$. tritici-repentis (parental form 113/00i-4 and lines 1-16i, 6-16i, 9-16i). In addition, the stress resistance of lines in laboratory conditions at the early stages of ontogenesis to $\mathrm{NaCl}$ salinity and prolonged flooding of seeds with water was assessed. Lines 33-16i, 37-16i, 32-16i and 9-16i showed a high ability to withstand excess moisture. Lines 33$16 i, 37-16 i, 32-16 i$ and 3-16i were characterized by high salt tolerance, exceeding the average value of $49.7 \%$. The listed samples are of interest as sources of anaerobic and salt stress, as well as donors of resistance to a group of fungal diseases: leaf and stem rust and tan spot. We associate the increased level of resistance of the new initial material with the presence of alien translocations in the original parental forms that participated in the origin of the lines. 